\title{
Susceptibilidad a la radiación ultravioleta-B del dinoflagelado Alexandrium catenella Kofoid Balech y de la diatomea Phaeodactylum tricornutum Bohlin
}

\author{
Susceptibility to ultraviolet-B radiation of the dinoflagellate Alexandrium catenella \\ Kofoid Balech, and the diatom Phaeodactylum tricornutum Bohlin
}

GINGER MARTINEZ, XIMENA MOLINA, MARIA ISABEL OLMEDO \& VIVIAN MONTECINO

Departamento de Ciencias Ecológicas, Facultad de Ciencias, Universidad de Chile, Casilla 653, Santiago, Chile, e-mail: bioptica@abello.dic.uchile.cl

\begin{abstract}
RESUMEN
La susceptibilidad diferencial de microalgas a la radiación UV-B (RUV-B, $280-320 \mathrm{~nm}$ ) genera patrones de predominio numérico dentro de los ensambles del fitoplancton. Sin embargo, a pesar que algunos eventos de floraciones algales nocivas (FAN) del dinoflagelado Alexandrium catenella han sido coincidentes con episodios de alta RUV-B en el extremo sur de Chile, se desconoce alguna relación de causalidad entre estos procesos. A través de los parámetros poblacionales tasa intrínseca de crecimiento $(\mu)$ y capacidad de carga $(K)$, se determinó el efecto de la RUV-B sobre la dinámica poblacional de cultivos de $A$. catenella, la que fue comparada con la respuesta demográfica de cultivos de una diatomea susceptible a la RUV-B, Phaeodactylum tricornutum. Los resultados mostraron que ambas especies presentaron una disminución significativa de $\mu$ frente a un incremento de RUV-B. Sin embargo, mientras que la respuesta de $A$. catenella mostró una dosis umbral para el crecimiento, bajo la cual se obtuvo una disminución de $\mu$ hasta $0,03 \mathrm{~d}^{-1}, P$. tricornutum presentó un decrecimiento proporcional, hasta un mínimo de $0,34 \mathrm{~d}^{-1}$. A pesar que el parámetro $\mathrm{K}$ en ambas especies presentó un decrecimiento similar frente a un gradiente de RUV-B, $A$. catenella mostró una significativa inhibición a partir de $2,9 \mathrm{KJ} \mathrm{m}^{-2} \mathrm{~d}^{-1}$, a diferencia de $P$. tricornutum, donde $\mathrm{K}$ se afectó a dosis iguales o mayores a $4,1 \mathrm{KJ} \mathrm{m}^{-2} \mathrm{~d}^{-1}$. Contrario a la predicción, los resultados indican una mayor susceptibilidad en $A$. catenella que en $P$. tricornutum al aumento de dosis de RUV-B, lo cual otorga una débil causalidad a este factor en lá determinación del patrón de predominio numérico presentado por $A$. catenella durante los eventos de FAN.
\end{abstract}

Palabras clave: RUV-B, Alexandrium catenella, Phaeodactylum tricornutum, crecimiento, capacidad de carga.

\begin{abstract}
Differential susceptibility to ultraviolet B (UV-B, 280 - $320 \mathrm{~nm}$ ) radiation among microalgae generates patterns of dominance in phytoplankton assemblages. However, despite some events of harmful algal blooms (HAB's) of the dinoflagellate Alexandrium catenella have been coincident with high UV-B radiation episodes in the southern region of Chile, a causal relationship has not been found among these processes. Through the population parameters, intrinsic growth rate $(\mu)$ and carrying capacity $(\mathrm{K})$, the UV-B effect over the population dynamic of $A$. catenella cultures was determined, and compared with the demographic response of cultures of the UV-B-susceptible diatom, Phaeodactylum tricornutum. Results showed that both species exhibit a significant decrease in $\mu$ faced with an increased UV-B radiation. Nevertheless, while response of $A$. catenella exhibited a threshold dose after which growth decreased down to $0.03 \mathrm{~d}^{-1}, \mu$-values in $P$. tricornutum showed a proportional decrease down to $0.34 \mathrm{~d}^{-1}$. Notwithstanding, the parameter $\mathrm{K}$ showed a similar decline in both species under a RUV-B gradient, $A$. catenella exhibited a significant inhibition at $2.9 \mathrm{KJ} \mathrm{m}^{-2} \mathrm{~d}^{-1}$, meanwhile in $P$. tricornutum $\mathrm{K}$ was affected under doses equal to or higher than $4.1 \mathrm{KJ} \mathrm{m}^{-2} \mathrm{~d}^{-1}$. Contrary to prediction, results indicated that with an increase in the UV-B radiation dose, $A$. catenella presented a higher susceptibility compared with $P$. tricornutum, granting a week causality to this factor in determining the dominance pattern of $A$. catenella during HAB's.
\end{abstract}

Key words: UV-B radiation, Alexandrium catenella, Phaeodactylum tricornutum, growth, carrying capacity. 


\section{INTRODUCCION}

La radiación ultravioleta - B (RUV-B, 280 - 320 $\mathrm{nm}$, según Neale 1999) produce efectos adversos en los ensambles biológicos (Lorenzen 1979, Smith et al. 1992, Cullen \& Neale 1993, Häder 1997), entre los cuales, el fitoplancton ha recibido especial atención debido a su condición autotrófica y por su posición basal en las cadenas alimentarias de los ecosistemas acuáticos (Worrest 1989, Karentz 1991, Bothwell et al. 1994).

Como parte de una respuesta global del fitoplancton frente a altas dosis de RUV-B, las especies muestran una conducta variable que difiere en función de numerosos atributos individuales, entre los cuales, el tamaño corporal ha sido descrito como una variable predictora de sensibilidad a la RUV-B (García-Pichel 1994, Häder et al. 1995, Jeffrey et al. 1996). Sin embargo, contrario a la hipótesis de dependencia del tamaño, existe numerosa evidencia que indica que diferencias interespecíficas en mecanismos y procesos ecofisiológicos estarían determinando la inhibición diferencial entre microalgas (Quesada \& Vincent 1997, Sundbäck et al. 1997, Laurion \& Vincent 1998, Wängberg et al. 1999). Además de aquellos estudios que demuestran que taxa de diatomeas presentan mayor tolerancia a la exposición de RUV-B que taxa de dinoflagelados (Jokiel \& York 1984, Ekelund 1990, Karentz 1994, Wängberg et al. 1996), una susceptibilidad diferencial a la RUV-B también ha sido encontrada entre diatomeas (Davidson et al. 1994, Davidson \& Marchant 1994) y entre dinoflagelados (Ekelund 1991), lo cual representa un fuerte argumento en torno a que diferencias taxonómicas por sobre atributos individuales podrían explicar la respuesta de las especies frente a incrementos en dosis de RUV-B. Si la RUV-B constituye un factor relevante en determinar patrones de coexistencia interespecífica entre microalgas, entonces aquellos taxa que muestran un predominio numérico durante condiciones de alta RUV-B, debieran presentar mayor tolerancia que aquellas especies que no proliferan bajo estas condiciones de radiación.

En la región austral de Chile, particularmente en la zona de fiordos y canales, el dinoflagelado Alexandrium catenella ha presentado aumentos demográficos explosivos y patrones de predominio numérico, procesos conocidos como Floraciones Algales Nocivas (FAN) (Guzmán et al. 1975, Guzmán \& Campodónico 1978, Muñoz \& Avaria 1997). A pesar que algunos estudios revelan un control interno de la generación de FAN, producido por interacciones generadas dentro de los ensambles (Iwasaki 1979, Arzul et al. 1999) o por características del ciclo de vida de A. catenella (Guzmán 1976), la mayoría de las evidencias apunta al control por factores causales externos, de origen abiótico como: irradiancia (Holligan 1987, Smayda 1997), condiciones hidrográficas (Lembeye et al. 1975), salinidad y temperatura ${ }^{1}$ y concentración de diferentes fuentes de nitróge$\mathrm{no}^{2}$, lo que ha recibido considerable respaldo empírico. Entre las condiciones de irradiancia, la RUV-B como factor causal de FAN aún no ha sido explorada, ello a pesar que periodos de incremento en dosis de RUV-B (Frederick et al. 1993) han coincidido con la descripción de episodios de FAN de A. catenella en la zona de Magallanes (Muñoz \& Avaria 1997).

Si la RUV-B tuviera algún poder explicativo de los patrones de predominio numérico mostrados por A. catenella durante los eventos de FAN, esta especie debería presentar un mayor grado de tolerancia a la RUV-B que aquellas que no proliferan bajo estas condiciones de radiación. En este estudio se determinó la dinámica poblacional de $A$. catenella frente a un rango de RUV-B, la que fue comparada con la conducta demográfica presentada por Phaeodactylum tricornutum Bohlin, diatomea cosmopolita y reconocida como un taxa susceptible a la RUV-B (Jokiel \& York 1984, Behrenfeld et al. 1992), y que además no se encuentra citada entre aquellos taxa formadores de FAN en Chile (Muñoz \& Avaria 1997).

\section{MATERIALES Y METODOS}

Cultivos unialgales de las cepas $\mathrm{ACC} 01^{3}$ de $A$. catenella Kofoid Balech y MLB292 ${ }^{4}$ de $P$. tricornutum Bohlin fueron mantenidos en fase exponencial de crecimiento durante $2-3$ meses bajo condiciones controladas de temperatura ( 15 $\pm 2{ }^{\circ} \mathrm{C}$ ) y fotoperiodo ( $\left.\mathrm{L}: \mathrm{O}=16: 8\right)$ en medio de cultivo estéril F/2 (Guillard 1975). Inóculos di-

\footnotetext{
' SEGUEL M, H LOYOLA \& M NUÑEZ (1995) Toxinas marinas. Sexto informe técnico, Proyecto FONDEF. ${ }^{2}$ PEREZ M \& M SEGUEL (1996) Efecto de distintas concentraciones y fuentes de nitrógeno sobre el crecimiento y toxicidad de Alexandrium catenella. Resúmenes VI Symposium de Algas Marinas Chilenas y IV Encuentro de Microalgólogos. Instituto de Fomento Pesquero, Puerto Montt, Chile, página 116.

${ }^{3}$ Inóculos provenientes del laboratorio de Benjamín Suárez en la Universidad de Chile.

${ }^{4}$ Inóculos provenientes del laboratorio de Gloria Collantes en la Universidad de Valparaiso.
} 
TABLA 1

Rangos de porcentajes de UV-B/RFA, UV-A/RFA y UV-B/UV-A, ensayadas en los cultivos de Alexandrium catenella y Phaeodactylum tricornutum. UV-B corresponde a la radiación ultravioleta - B $(280-320 \mathrm{~nm})$, UV-A a la radiación ultravioleta - A $(320-400 \mathrm{~nm})$ y RFA a la radiación fotosintéticamente activa $(400-700 \mathrm{~nm})$. Se incluyen valores promedios de proporciones obtenidas en condiciones naturales (V. Montecino, datos no publicados)

Ranges of percentages of UV-A/RFA, UV-B/RFA and UV-B/UV-A incident over Alexandrium catenella and Phaeodactylum tricornutum cultures. UV-B is ultraviolet-B radiation $(280-320 \mathrm{~nm})$, UV-A is ultraviolet-A radiation $(320-400 \mathrm{~nm})$ and $\mathrm{RFA}(=\mathrm{PAR})$ is Photosynthetically Active Radiation $(400-700 \mathrm{~nm})$. Mean values of these proportions obtained in natural conditions are included (V. Montecino, unpublished data)

\begin{tabular}{lccc}
\hline & $\frac{\mathrm{UV}-\mathrm{B}}{\mathrm{RFA}}$ & $\frac{\mathrm{UV}-\mathrm{A}}{\mathrm{RFA}}$ & $\underline{\mathrm{UV}-\mathrm{B}}$ \\
\hline A. catenella & $\mathbf{0 , 0 3 - 0 , 6 0}$ & $2,2-18,1$ & $\mathrm{UV}-\mathrm{A}$ \\
\hline . tricornutum & $\mathbf{0 , 0 7 - 0 , 3 2}$ & $12,5-29,9$ & $0,27-3,6$ \\
Condición natural & 0,40 & 11,0 & $0,20-1,5$ \\
\hline
\end{tabular}

luidos de los cultivos fueron transferidos a una cámara de incubación en bolsas de polietileno de $100 \mathrm{ml}$, transparentes a la RUV-B (Nasco WHIRLPack $^{\oplus}$ ) y aclimatados a las condiciones experimentales de irradiancia durante $72 \mathrm{~h}$ para $A$. catenella y $48 \mathrm{~h}$ para $P$. tricornutum, periodos definidos en función de variaciones no significativas de la concentración de pigmentos fotosintéticos (clorofila a).

Cultivos controles y tratamientos fueron expuestos a $112,7 \pm 2,5 \mu$ moles $\mathrm{m}^{-2} \mathrm{~s}^{-1}$ de RFA (radiación fotosintéticamente activa, $400-700 \mathrm{~nm}$ ) provistos por tubos fluorescentes PHILIPS, TLD $18 \mathrm{~W} / 33$ y a un rango de radiación UV-A (RUV-A,

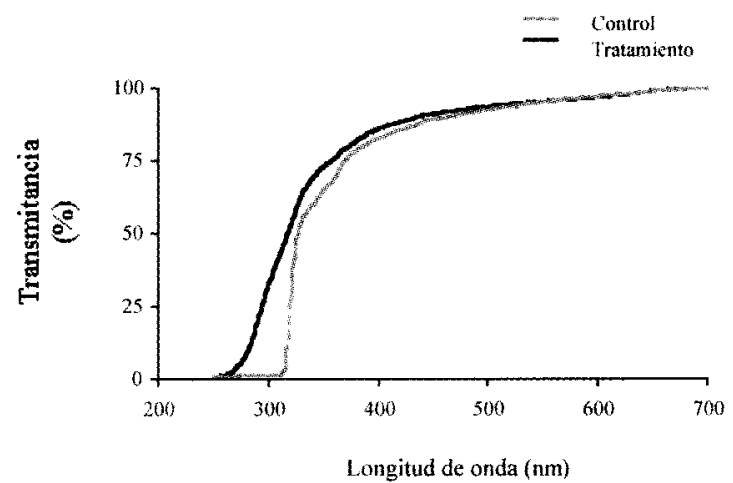

Fig. 1. Espectros de transmitancia de los diferentes materiales utilizados como filtros durante la implementación de los experimentos. poliester + bolsa (Control) y acetato de celulosa + bolsa (Tratamiento).

Transmitance spectra of the differents materials used as filters in the experiments. polyester + bag (Control) and - cellulose acetate + bag (Treatment).
$365 \mathrm{~nm}$ ) mediante tubos PHILIPS, TLD 18W/08, cuyas proporciones se indican en la Tabla 1 . Un gradiente de radiación UV-B (RUV-B, $313 \mathrm{~nm}$ ) provisto por tubos Q Panel® Co. U.S.A, fue aplicado sobre los cultivos tratamientos manipulando la distancia a la fuente de emisión y el tiempo de exposición (Tabla 2). El rango de RUV-B fue establecido en función de mediciones de RFA, UV-A y UV-B similares a las obtenidas en condiciones naturales (Tabla 1). Tres réplicas de los controles ( $\sin$ RUV-B) fueron aisladas de la RUVB y RUV-C mediante filtros de poliester ( $>330$ nm) y un número igual de réplicas de los tratamientos (con RUV-B) fueron envueltas en filtros de acetato de celulosa para impedir su exposición a la RUV-C (Fig. 1).

Inóculos de $1 \mathrm{ml}$ fueron extraídos en condiciones axénicas desde los cultivos de $A$. catenella durante 14 días y cada 72 horas, los que fueron fijados con formaldehído al $5 \%$ para recuento celular en una cámara Sedgwick-Rafter. Los cultivos de $P$. tricornutum fueron mantenidos entre 7 - 8 días, a los que diariamente se les removió y fijó inóculos de $1 \mathrm{ml}$ para realizar el recuento de células en hemocitómetro bajo un microscopio de contraste de fases (Leitz Wetzlar). En cada réplica se obtuvo la tasa intrínseca de crecimiento poblacional $(\mu)$ a través de la siguiente expresión:

$$
\mu=\left(\operatorname{lnC}_{\mathrm{f}}-\ln \mathrm{C}_{\mathrm{i}}\right) \cdot\left(\mathrm{t}_{\mathrm{f}}-\mathrm{t}_{\mathrm{i}}\right)^{-1}\left(\mathrm{~d}^{-1}\right)
$$

donde, $\mathrm{C}_{\mathrm{f}}$ es la concentración dẹ células en el tiempo final $\left(\mathrm{t}_{\mathrm{f}}\right)$ y $\mathrm{C}_{\mathrm{i}}$ la concentración de células en el tiempo inicial $\left(t_{i}\right)$ de la etapa exponencial de

\footnotetext{
${ }^{5}$ Dosis no ponderada
} 
crecimiento (Kain 1987). También se determinó la capacidad de carga (K) de cada cultivo como la densidad máxima de células alcanzada en cada bolsa experimental.

Para analizar los resultados obtenidos de experimentos realizados en forma sucesiva, se estandarizaron las dosis de RUV-B obtenidas en cada tratamiento por las dosis de RFA y RUV-A incidentes en cada condición control, mediante el siguiente factor de corrección:

$$
F_{c}=\left(R F A_{T}+R U V-A_{T}\right) /\left(R F A_{C}+R U V-A_{C}\right)
$$

donde, los subíndices $\mathrm{T}$ y $\mathrm{C}$ representan las condiciones tratamiento y control respectivamente.

Para comparar la respuesta de ambas especies frente a un gradiente de dosis de RUV-B, los parámetros $\mu$ y $\mathrm{K}$ fueron estandarizados por el valor obtenido en cada condición control, de modo que $\mu^{*}=\mu / \mu_{\text {control }}$ y $K^{*}=K / K_{\text {control }}$.

Las comparaciones entre tratamientos y controles se realizaron mediante la prueba no paramétrica Mann-Whitney. Mediante un análisis de regresión múltiple se estimó la influencia del factor tiempo de exposición en la respuesta de $\mu$ y $K$ frente a la RUV-B (Hair et al. 1995). Los valores de $\mu^{*}$ y $K^{*}$ frente a un gradiente de RUV-B fueron ajustados a una curva teórica mediante un análisis de regresión simple y sus pendientes fueron comparadas a través de un análisis de covarianza ANCOVA (Sokal \& Rohlf 1981).

\section{RESULTADOS}

\section{Tasa de crecimiento $(\mu)$}

El parámetro $\mu$ en ambas especies fue afectado en forma significativa por el aumento de dosis de RUV-B. En dosis de RUV-B menores o iguales a $2,4 \mathrm{KJ} \mathrm{m}^{-2} \mathrm{~d}^{-1}$, los valores de $\mu$ en $A$. catenella fluctuaron entre $0,23 \pm 0,03 \mathrm{~d}^{-1}$ y $0,25 \pm 0,04 \mathrm{~d}^{-1}$, los que no difirieron del valor de la situación control (Mann-Whitney U-test: $\mathrm{U}=17,5 ; \mathrm{P}=$ 0,$94 ; \mathrm{n}=6$; Tabla 2). Sin embargo, al aumentar la dosis de RUV-B sobre $2,9 \mathrm{KJ} \mathrm{m}^{-2} \mathrm{~d}^{-1}$, A. catenella presentó un decrecimiento en el valor de $\mu$, entre un 56 y $86 \%$ respecto del control (Mann-Whitney U-test: $\mathrm{U}=0,0 ; \mathrm{P}<0,001 ; \mathrm{n}=11$; Tabla 2). Dentro del rango de susceptibilidad a la RUV-B, el valor mínimo de $\mu$ detectado en $A$. catenella fue de $0,03 \mathrm{~d}^{-1}$, obtenido con $2,9 \mathrm{KJ} \mathrm{m}^{-2} \mathrm{~d}^{-1}$, el cual se mantuvo relativamente constante hasta dosis de $9,4 \mathrm{KJ} \mathrm{m}^{-2} \mathrm{~d}^{-1}$.

Por el contrario, dosis de RUV-B menores a 4,1 $\mathrm{KJ} \mathrm{m}^{-2} \mathrm{~d}^{-1}$ con una hora de exposición no inhibieron los valores de $\mu$ en $P$. tricornutum. A partir de esta dosis y con dos horas de exposición a la RUV-B, se detectó una disminución significativa de $\mu$ hasta $0,41 \mathrm{~d}^{-1}$ (Mann-Whitney U-test: $\mathrm{U}=$ 0,$0 ; \mathbf{P}=0,046 ; \mathbf{n}=3$; Tabla 2). Dosis mayores condujeron a una progresiva disminución de $\mu$, que varió entre 25 y $79 \%$, respecto de los valores detectados en la condición control (Mann-Whitney U-test: $\mathrm{U}=2,0 ; \mathrm{P}<0,001 ; \mathrm{n}=11$; Tabla 2). A pesar que $P$. tricornutum se expuso frente a dosis significativamente mayores a aquellas aplicadas a $A$. catenella, con un valor máximo de $16,3 \mathrm{KJ}$ $\mathrm{m}^{-2} \mathrm{~d}^{-1}$ con 4 horas de exposición, los valores de $\mu$ en $P$. tricornutum se mantuvieron en $0,34 \mathrm{~d}^{-1}$ (Tabla 2).
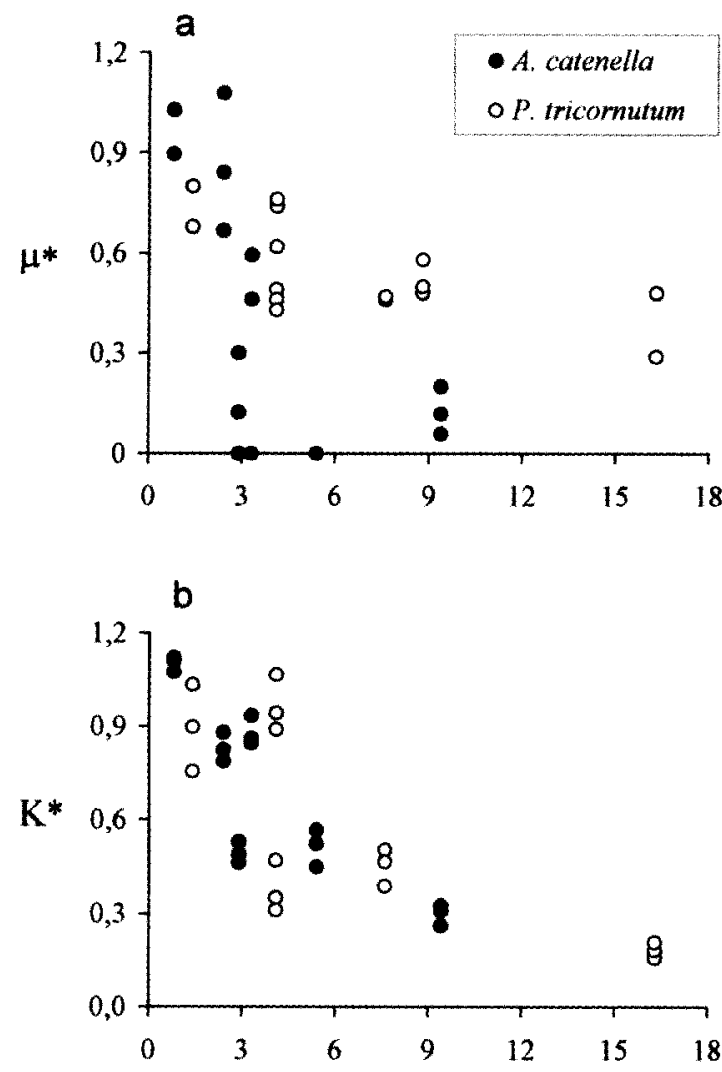

Dosis de RUV-B $\left(\mathrm{KJ} \mathrm{m}^{-2} \mathrm{~d}^{-1}\right)$

Fig. 2. Respuesta de los parámetros tasa de crecimiento $\left(\mu^{*}\right)$ y capacidad de carga $\left(K^{*}\right)$ estandarizados por los valores estimados en la condición control, de Alexandrium catenella $\bullet$ Phaeodactylum tricornutum $\mathrm{O}$, frente a un gradiente de radiación UV-B.

Response of the parameters growth rate $\left(\mu^{*}\right)$ and carrying capacity $\left(\mathrm{K}^{*}\right)$ standarized by the values obtained in the control condition, in Alexandrium catenella $\bullet$ and Phaeodactylum tricornutum $\mathrm{O}$, under a gradient of UV-B radiation. 
Los valores de $\mu$ estandarizados por la condición control $\left(\mu^{*}\right)$ en ambas especies se ajustaron en forma significativa a un modelo logarítmico (A. catenella: $\mathrm{R}^{2}=0,64 ; \mathrm{P}<0,001 ; \mathrm{n}=13, \mathrm{y} P$. tricornutum: $\mathrm{R}^{2}=0,55 ; \mathrm{n}=17 ; \mathrm{P}<0,001$, Fig. $\left.2 \mathrm{a}\right)$. La curva de respuesta de $A$. catenella frente a la RUV-B presentó una mayor pendiente $(\mathrm{m})$ que la curva de respuesta de $P$. tricornutum (ANCOVA, $\mathrm{F}_{(1,28)}=6,03 ; \mathrm{P}=0,02 ; \mathrm{m}_{\text {A. catenella }}=-0,070 \pm 0,02 ;$ $\left.\mathrm{m}_{P \text {. tricornutum }}=-0,02 \pm 0,01\right)$, revelando con ello una mayor susceptibilidad a la RUV-B de A. catenella que $P$. tricornutum (Fig. 2a). La curva de respuesta de $A$. catenella frente al gradiente de RUV-B mostró una región en la que leves cambios en dosis produjeron significativas variaciones de $\mu^{*}$ (Fig. 2a), lo que indica que $2,9 \mathrm{KJ} \mathrm{m}^{-2} \mathrm{~d}^{-1}$ de RUV-B constituiría una dosis umbral para el crecimiento de A. catenella. Por el contrario, los valores de $\mu^{*}$ en $P$. tricornutum no mostraron un marcado decrecimiento, sino que disminuyeron en forma proporcional frente a un aumento de RUV-B (Fig. 2a).
El análisis entre los factores dosis y tiempo de exposición reveló que este último explicó en mayor grado la disminución de $\mu$ en $P$. tricornutum (ANOVA, $F_{(2,3)}=6,6 ; P=0,08 ; b_{\text {dosis }}=-0,079 ; \mathrm{P}=$ 0,$\left.78 ; B_{\text {tiempo }}=-0,92 ; \mathrm{P}=0,039\right)$. Mientras que $A$. catenella se afectó con dosis de exposición a la RUV-B mayores a 2 horas, $P$. tricornutum mantuvo la tasa de crecimiento entre valores de 0,34 hasta $0,09 \mathrm{~d}^{-1}$ con exposiciones de cuatro y seis horas, respectivamente (Tabla 2 ).

\section{Capacidad de carga $(K)$}

El parámetro $\mathrm{K}$ en ambas especies fue afectado por dosis crecientes de RUV-B. Los valores de K en $A$. catenella no difirieron de los valores presentados en la condición control en dosis de 0,8 y $2,4 \mathrm{KJ} \mathrm{m}^{-2} \mathrm{~d}^{-1}$, con dos horas de exposición (MannWhitney U-test: $\mathrm{U}=15,0 ; \mathrm{P}=1,0 ; \mathrm{n}=6$; Tabla 2). Sin embargo, en forma similar a lo encontrado

TABLA 2

Tasa de crecimiento $(\mu)$ y capacidad de carga (K) de Alexandrium catenella y Phaeodactylum tricornutum frente a un rango de dosis de radiación UV-B (RUV-B). Se indican los tiempos de exposición a la radiación UV-B $\left(\mathrm{T}_{\mathrm{exp}}\right)$. Los valores de $\mu$ y $\mathrm{K}$ representan el promedio \pm 1 EE de tres réplicas. Las comparaciones de los valores en las condiciones control y tratamiento fueron realizadas mediante la prueba no paramétrica Mann-Whitney. El intervalo de valores de $\mu$ y $\mathrm{K}$ para A. catenella estimados en la condición control fue $0,14-0,39 \mathrm{~d}^{-1}$ y $1860-14767$ cels . $\mathrm{ml}^{-1}$ respectivamente, y para $P$. tricornutum $0,41-0,93 \mathrm{~d}^{-1}$ y $1632-3400 \cdot 10^{3} \mathrm{cels}^{-\mathrm{ml}^{-1}}$ respectivamente. $* \mathrm{P} \leq 0,05$

Growth rate $(\mu)$ and carrying capacity $(\mathrm{K})$ of Alexandrium catenella and Phaeodactylum tricornutum under a range of doses of UV-B radiation (RUV-B). Exposition times to UV-B radiation ( $T_{\text {exp }}$ ) are depicted. $\mu$ and $K$ values represent a mean $\pm 1 \mathrm{SE}$ from three replicates. Contrasts between values attained in treatment and control conditions were assessed by using the non-parametric test Mann-Whitney. Ranges of $\mu$ and $\mathrm{K}$ values in $A$. catenella obtained in control were $0.14-0.39 \mathrm{~d}^{-1}$ and $1860-14767$ cells $\cdot \mathrm{ml}^{-1}$ respectively, and for $P$. tricornutum $0.41-0.93 \mathrm{~d}^{-1}$ and $1632-3400 \cdot 10^{3}$ cells $\cdot \mathrm{ml}^{-1}$ respectively. $* \mathrm{P} \leq 0.05$

\begin{tabular}{|c|c|c|c|c|c|c|c|}
\hline \multirow{2}{*}{$\begin{array}{l}\text { Especie } \\
\text { A. catenella }\end{array}$} & \multirow{2}{*}{ 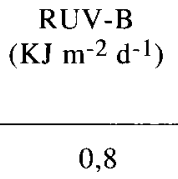 } & \multirow{2}{*}{$\begin{array}{c}T_{\exp } \\
(\mathrm{h}) \\
2\end{array}$} & \multicolumn{2}{|r|}{$\begin{array}{c}\mu \\
\left(d^{-1}\right)\end{array}$} & \multicolumn{3}{|c|}{$\begin{array}{c}\mathrm{K} \\
\left(\mathrm{cels} \cdot \mathrm{ml}^{-1}\right) \\
10^{3}\end{array}$} \\
\hline & & & 0,25 & $\pm 0,040$ & 11,8 & \pm & 0,15 \\
\hline & 2,4 & 2 & 0,23 & $\pm 0,030$ & 2,7 & \pm & 0,09 \\
\hline & 2,9 & 2 & 0,03 & $\pm 0,020^{*}$ & 5,2 & \pm & $0,21^{*}$ \\
\hline & 3,3 & 2 & 0,07 & $\pm 0,007$ & 1,8 & \pm & $0,06^{*}$ \\
\hline & 5,4 & 2 & 0,03 & $\pm 0,003^{*}$ & 1,0 & \pm & $0,07 *$ \\
\hline & 9,4 & 2 & 0,04 & $\pm 0,011^{*}$ & 1,0 & \pm & $0,06^{*}$ \\
\hline \multirow[t]{6}{*}{ P. tricornutum } & 1,4 & 2 & 0,63 & $\pm 0,035$ & 2900 & \pm 2 & 60 \\
\hline & 4,1 & 1 & 0,41 & $\pm 0,052 *$ & 1860 & \pm & 37 \\
\hline & 4,1 & 2 & 0,41 & $\pm 0,011 *$ & 1200 & \pm 1 & $51 *$ \\
\hline & 7,6 & 6 & 0,09 & $\pm 0,004^{*}$ & & - & \\
\hline & 8,8 & 2 & 0,34 & $\pm 0,020^{*}$ & 900 & \pm & $72 *$ \\
\hline & 16,3 & 4 & 0,34 & $\pm 0,030^{*}$ & 73 & $\pm ?$ & $37^{*}$ \\
\hline
\end{tabular}

\footnotetext{
- no determinado
} 
con el parámetro $\mu$, a partir de $2,9 \mathrm{KJ} \mathrm{m}^{-2} \mathrm{~d}^{-1}$, con dos horas de exposición a la RUV-B, los valores de $\mathrm{K}$ disminuyeron en forma significativa entre un 53 y $99 \%$, respecto de la situación control (Mann-Whitney U-test: $\mathrm{U}=28,0 ; \mathrm{P}=0,019 ; \mathrm{n}=$ 12; Tabla 2). A pesar que los niveles de $\mathrm{K}$ en $A$. catenella disminuyeron en forma proporcional al incremento de RUV-B, en dosis de RUV-B mayores a $3,3 \mathrm{KJ} \mathrm{m}^{-2} \mathrm{~d}^{-1}$, los valores de $\mathrm{K}$ se mantuvieron relativamente constantes, en $1 \cdot 10^{3} \mathrm{cels} \cdot \mathrm{ml}^{-1}$ (Tabla 2).

El valor de $\mathrm{K}$ en $P$. tricornutum no disminuyó en dosis menores o iguales a $4,1 \mathrm{KJ} \mathrm{m}^{-2} \mathrm{~d}^{-1}$, con una hora de exposición a la RUV-B (MannWhitney U-test: $\mathrm{U}=15,0 ; \mathrm{P}=0,631 ; \mathrm{n}=6$; Tabla 2), sin embargo, al aumentar el tiempo de exposición a dos horas, se detectó un decrecimiento progresivo significativo frente a mayores dosis de RUV-B (Mann-Whitney U-test: $\mathrm{U}=0,0 ; \mathrm{P}<$ 0,001; $\mathrm{n}=$ 9; Tabla 2).

Los valores de $\mathrm{K}$ estandarizados $\left(\mathrm{K}^{*}\right)$ en $A$. catenella y $P$. tricornutum frente a un gradiente de RUV-B se ajustaron a un modelo logarítmico (A. catenella: $\mathrm{R}^{2}=0,69 ; \mathrm{P}<0,0001 ; \mathrm{n}=18$, y $P$. tricornutum: $\mathrm{R}^{2}=0,62 ; \mathrm{n}=15 ; \mathrm{P}<0,001$, Fig. 2b). El análisis de pendientes reveló que las curvas de respuesta de ambas especies frente a la RUV-B no difirieron entre sí (Fig. 2b, ANCOVA; $\left.\mathrm{F}_{(1,29)}=30,6 ; \mathrm{P}=0,10\right)$, detectándose en $A$. catenella una pendiente de $-0,082(\mathrm{EE}=0,013) \mathrm{y}$ en $P$. tricornutum una pendiente de $-0,045$ (EE $=$ 0,011 ) (Fig. 2b).

\section{DISCUSION}

A pesar que ambas especies presentaron una disminución significativa de los parámetros $\mu$ y K frente a un incremento de RUV-B, no se cumplió la predicción de mayor tolerancia a la RUV-B por $A$. catenella en relación con $P$. tricornutum. Respecto a otros grupos de microalgas, las especies de dinoflagelados han sido descritas como taxa susceptibles a la RUV-B, principalmente comparados con taxa de diatomeas, tanto en condiciones naturales (Jokiel \& York 1984) como artificiales (Ekelund 1990). Mientras que dosis entre 0,08 y $1,5 \mathrm{KJ} \mathrm{m}^{-2} \mathrm{~d}^{-1}$ (Ekelund 1990, Ekelund 1991) inhiben la tasa de crecimiento de dinoflagelados, solo a partir de $6,8 \mathrm{KJ} \mathrm{m}^{-2} \mathrm{~d}^{-1}$ se ha obtenido una disminución en la tasa de crecimiento y en el porcentaje de sobrevivencia de diatomeas (Davidson et al. 1994, Peletier et al. 1996).

Si bien, A. catenella aparece como más susceptible a la RUV-B que $P$. tricornutum, este patrón cambia diametralmente cuando se la compara con otras especies de dinoflagelados. En condiciones experimentales similares a las utilizadas en este trabajo, los dinoflagelados Gyrodinium aureolum, Prorocentrum minimun y Heterocapsa triquetra presentan inhibición en sus tasas de crecimiento a partir de dosis de $1,5 \mathrm{KJ} \mathrm{m}^{-2} \mathrm{~d}^{-1}$, con 1 hora diaria de exposición a la RUV-B (Ekelund 1991), lo cual es significativamente inferior a la dosis umbral detectada aquí para el crecimiento de $A$. catenella, de $2,9 \mathrm{KJ} \mathrm{m}^{-2} \mathrm{~d}^{-1}$ con 2 horas de exposición. Estas diferencias revelarían que $A$. catenella podría considerarse como una de las especies con mayor capacidad de tolerar aumentos de RUV-B entre los dinoflagelados, lo cual le otorgaría ventajas comparativas bajo condiciones de alta RUV-B.

La susceptibilidad diferencial a la RUV-B encontrada entre dinoflagelados y diatomeas estaría también relacionada con diferencias en tolerancia a bajos niveles de RFA. Debido a que los dinoflagelados formadores de proliferaciones algales, entre ellos A. catenella, presentan mayor adaptación a condiciones de baja RFA, estas especies se verían comparativamente más afectadas a la exposición de RUV-B que aquellas que no describen tales episodios (Holligan 1987). Debido a que los mecanismos compensatorios a los efectos adversos de la RUV-B son gatillados por la RFA y la RUV-A (Roy 1999), los dinoflagelados presentarían menor eficiencia en los mecanismos de fotoprotección frente a la RUV-B, en contraposición a $P$. tricornutum, especie descrita como tolerante a la RUV-A (Jokiel \& York 1984) y que incluso habita en ambientes someros de la zona intermareal costera (G. Collantes, comunicación personal).

Debido a que los valores máximos de RUV-B medidos en condiciones naturales superan en forma significativa la dosis umbral $\left(2,9 \mathrm{KJ} \mathrm{m}^{-2} \mathrm{~d}^{-1}\right)$ para el crecimiento de $A$. catenella, este especie se encontraría en condiciones de desventaja respecto a otras especies del fitoplancton con las que coexiste, bajo un clima lumínico de alta dosis de RUV-B. Estos resultados, aunados a la mayor tolerancia a la RUV-B presentada por la diatomea $P$. tricornutum, especie que no ha sido descrita formando parte de los ensambles de microalgas durante el desarrollo de FAN en la región austral del país (Muñoz \& Avaria 1997), otorgan débil causalidad al factor RUV-B en determinar el patrón de predominio numérico mostrado por $A$. catenella durante estos episodios. Considerando que no existe información acerca del efecto de la RUV-B sobre el crecimiento en cultivos de $A$. catenella, este trabajo constituye un aporte al conocimiento de la respuesta de esta especie frente a dosis crecientes de RUV-B, potencialmente relevante si se considera que condiciones de alta 
RUV-B son concurrentes con el desarrollo de FAN de A. catenella en el extremo austral de Chile.

\section{AGRADECIMIENTOS}

Los autores agradecen a Miriam Seguel del Instituto de Fomento Pesquero y a Benjamín Suárez de la Facultad de Medicina de la Universidad de Chile por proveer los cultivos de A. catenella y a Gloria Collantes del Instituto de Oceanología de la Universidad de Valparaíso, quién proporcionó los cultivos de $P$. tricornutum. También se agradece a Mónica Orellana, Gabriela Hannach y a dos correctores anónimos, quienes contribuyeron en forma sustancial a mejorar este trabajo. Este estudio fue financiado por el proyecto FONDECYT N ${ }^{\circ} 1960875$, concurrente al Programa FONDAP-HUMBOLDT.

\section{LITERATURA CITADA}

ARZUL G, M SEGUEL, L GUZMAN, E ERARD-LE DENN (1999) Comparison of allelopathic properties in three toxic Alexandrium species. Journal of Experimental Marine Biology and Ecology 232: 285-295.

BEHRENFELD MJ, JT HARDY \& H LEE II (1992) Chronic effects of ultraviolet-B radiation on growth and cell volume of Phaeodactylum tricornutum (Bacillariophyceae). Journal of Phycology 28: 757 760.

BOTHWELL ML, D SHERBOT \& CM POLLOCK (1994) Ecosystem response to solar ultraviolet-B radiation: influence of trophic-level interactions. Science 265 : 97-100.

CULLEN J \& P NEALE (1993) Quantifying the effects of ultraviolet radiation on aquatic photosynthesis. En: Yamamoto HY \& CM Smith (eds) Photosynthetic responses to the environment: 45-60. American Society of Plant Physiologists, Rockwille, MD.

DAVIDSON AT \& HJ MARCHANT (1994) Comparative impact of in situ UV exposure on productivity, growth and survival of antarctic Phaeocystis and diatoms. Polar Biology 7: 53-69.

DAVIDSON AT, D BRAMICH, HJ MARCHANT \& A McMINN (1994) Effects of UV-B irradiation on growth and survival of antarctic marine diatoms. Marine Biology 119: 507-515.

EKELUND NG (1990) Effects of UV-B radiation on growth and motility of four phytoplankton species. Physiologia Plantarum 78: 590-594.

EKELUND NG (1991) The effects of UV-B radiation on dinoflagellates. Journal of Plant Physiology 138: 274278.

FREDERICK JE, PF SOULEN, SB DIAZ, L SMOLSKAIA, CR BOOTH, T LUCAS \& D NEUSCHULER (1993) Solar ultraviolet irradiance observed from southern Argentina: September 1990 to March 1991. Journal of Geophysical Research 98: 8891-8897.
GARCIA-PICHEL F (1994) A model for internal selfshading in planktonic organisms and its implications for the usefulness of ultraviolet sunscreens. Limnology and Oceanography 39: 1704-1717.

GUILLARD RL (1975) Culture of phytoplankton for feeding marine invertebrates. En: Smith WL \& MH Chanley (eds) Culture of marine invertebrates animals 29-60. Plenum Publishing, New York.

GUZMAN L (1976) Estudios sobre un florecimiento tóxico causado por Gonyaulax catenella en Magallanes. V. El posible cisto ecdísico de G. catenella. Anales del Instituto de la Patagonia, Punta Arenas (Chile) 7 201-206.

GUZMAN L \& I CAMPODONICO (1978) Mareas rojas en Chile. Interciencia 3: 144-149.

GUZMAN L, I CAMPODONICO \& J HERMOSILLA (1975) Estudios sobre un florecimento tóxico causado por Gonyaulax catenella en Magallanes. I. Distribución espacial y temporal de $G$. catenella. Anales del Instituto de la Patagonia, Punta Arenas (Chile) 6: 173-183.

HÄDER DP (1997) Vertical migration and distribution of primary producers in aquatic ecosystems - The effects of enhanced solar UVB. Photochemistry and Photobiology 65: 263-264.

HÄDER DP, RC WORREST, HD KUMAR \& RC SMITH (1995) Effects of increased solar ultraviolet radiation on aquatic ecosystems. Ambio 24: $174-180$.

HAIR JF Jr, RE ANDERSON, RL TATHAM \& WC BLACK (1995) Multivariate Data Analysis. Fourth edition Prentice Hall, New Jersey. xvii +745 pp.

HOLLIGAN PM (1987) The physical environment of exceptional phytoplankton blooms in the northeast Atlantic. Rapports et Proces Verbaux des Reunions Conseil International pour 1' Exploration de la Mer 187: 9-18.

IWASAKI H (1979) Physiological ecology of red tide flagellates. En: Levandowsky M \& S Hunter (eds) Biochemistry and Physiology of Protozoa: 357-393. Academic Press, California.

JEFFREY WH, P AAS, M MAILLE LYONS, RB COFFIN, RJ PLEDGER \& DL MITCHELL (1996) Ambient solar radiation induced photodamage in marine bacterioplankton. Photochemistry and Photobiology 64: $419-427$

JOKIEL PL \& RH YORK Jr (1984) Importance of ultraviolet radiation in photoinhibition of microalgal growth. Limnology and Oceanography 29: 192-199.

KAIN JM (1987) Seasonal growth and photoinhibition in Plocamium cartilagineum (Rhodophyta) off the Isle Man. Phycology 26: 88-99.

KARENTZ D (1991) Ecological considerations of antarctic ozone depletions. Antarctic Science 3: 3-11.

KARENTZ D (1994) Ultraviolet tolerance mechanisms in Antarctic marine organisms. En: Weiler CS \& PA Penhale (eds) Ultraviolet radiation in Antarctica: Measurements and biological effects: 93-110. American Geophysical Union, Washington.

LAURION I \& WF VINCENT (1998) Cell size versus taxonomic composition as determinants of UVsensitivity in natural phytoplankton communities. Limnology and Oceanography 43: 1774-1779. 
LEMBEYE G, L GUZMAN \& I CAMPODONICO (1975) Estudios sobre un florecimiento tóxico causado por Gonyaulax catenella en Magallanes. III. Fitoplancton asociado. Anales del Instituto de la Patagonia, Punta Arenas (Chile) 6: 197-208.

LORENZEN C (1979) Ultraviolet radiation and phytoplankton photosynthesis. Limnology and Oceanography 24: 1117-1120.

MUÑOZ P \& S AVARIA (1997) Fenómenos de marea roja y otras floraciones algales en Chile. Ciencia y Tecnología del Mar 20: 175-192.

NEALE P (1999) Spectral weighting functions for quantifying effects of ultraviolet radiation in marine ecosystems. En: de Mora S, S Demers \& M Vernet (eds) The effects of UV radiation in the marine environment. Cambridge University Press, New York.

PELETIER H, WWC GIESKES \& AGJ BUMA (1996) Ultraviolet-B radiation resistance of benthic diatoms isolated from tidal flats in the Dutch Wadden Sea. Marine Ecology Progress Series 135: 163-168.

QUESADA A \& WF VINCENT (1997) Strategies of adaptation by antarctic cyanobacteria to ultraviolet radiation. European Journal of Phycology 32: 335 342.

ROY S (1999) Strategies for the minimisation of UVinduced damage. En: de Mora S, S Demers \& M Vernet (eds) The effects of UV radiation in the marine environment. Cambridge University Press, New York.
SMAYDA TJ (1997) What is a bloom? A commentary. Limnology and Oceanography 42: 1132-1136.

SMITH RC, BB PREZELIN, KS BAKER, RR BIDIGARE, NP BOUCHER, T COLEY, D KARENTZ, S MaCINTYRE, HA MATLICK, D MENZIES, M ONDRUSEK, Z WAN \& KJ WATERS (1992) Ozone depletion: ultraviolet radiation and phytoplankton biology in Antarctic waters. Science 255: 952-958.

SOKAL R \& F ROHLF (1981) Biometry. Second edition. W H Freeman and Company, San Francisco. xviii + $859 \mathrm{pp}$.

SUNDBÄCK K, S ODMARK, A WULFF, C NILSSON \& SÅ WÄNGBERG (1997) Effects of enhanced UVB radiation on a marine benthic diatom mat. Marine Biology 128: 171-179.

WÄNGBERG SÅ, JS SELMER \& K GUSTAVSON (1996) Effects of UV-B radiation on biomass and composition in marine phytoplankton communities. Scientia Marina $60: 81-88$.

WÄNGBERG SA, K GARDE, K GUSTAVSON \& JS SELMER (1999) Effects of UV-B radiation on marine phytoplankton communities. Journal of Plankton Research 21: 147-166.

WORREST RC (1989) What are the effects of UV-B radiation on marine organisms? En: Schneider $T$ (ed) Atmospheric ozone research and its policy implications: 269-278. Elsevier, Amsterdam. 